\title{
The Relationship Between the Level Fabp4, Risks of Type 2 Diabetes Mellitus, and Cardiovascular Events
}

\author{
Yelena Laryushina ${ }^{1}$, Viktoriya Parakhina ${ }^{2 *}$, Anar Turmukhambetova ${ }^{3}$, Lyudmila Turgunova $^{1}$, Lyazat Ibraeva ${ }^{4}$, Dana Amirkhanova $^{1}$, \\ Farida Nildibayeva $^{1}$ \\ ${ }^{1}$ Department of the Internal Medicine No. 2, Karaganda Medical University, Karaganda, Kazakhstan; ${ }^{2}$ Department of Medicine, \\ Karaganda Medical University, Karaganda, Kazakhstan; ${ }^{3}$ Department of Strategic Development and Science, Karaganda \\ Medical University, Karaganda, Kazakhstan; ${ }^{4}$ Department of the Internal Medicine No. 3, Karaganda Medical University, \\ Karaganda, Kazakhstan
}

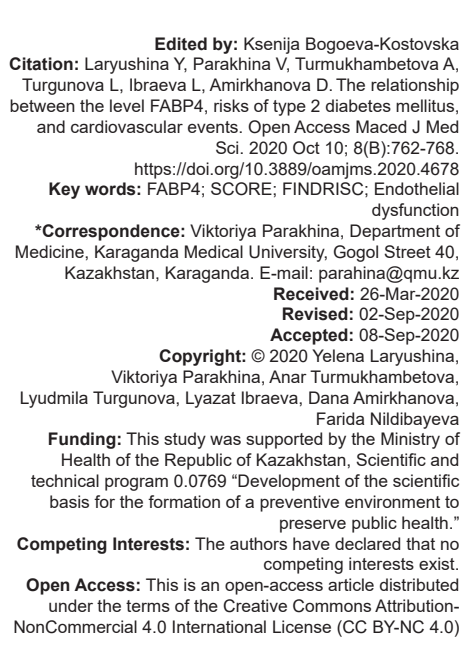

\section{Introduction}

Type 2 diabetes mellitus (T2DM) is one of the four priority non-communicable diseases. Measures are planned on prevention of diabetes, all over the world. During last few decades, the incidence and prevalence of diabetes have been increased dramatically. According to the International Diabetes Federation data in 2017 about 425 million of adults worldwide suffered from diabetes, compared to 108 million in 1980 [1]. The global diabetes incidence rate (standardized by age) has nearly doubled since 1980 , rising from $4.7 \%$ to $8.5 \%$ in the population. The growth of diabetes incidence in the Republic of Kazakhstan from 2004 to 2017 was $136.8 \%$ [2].

As well as high diabetes incidence, another 352.1 million of peopleworldwide havea preliminary diabetes stage called Impair Glucose Tolerance. The health-care specialists expected that the number of prediabetic persons will increase till 531.6 million in 2045 [1]. This epidemiological situation was resulted from to the growth T2DM modifiable risk factors, such as overweight or obesity, malnutrition, and low physical activity. In 2016, T2DM caused 1.6 million deaths worldwide. The hyperglycemia caused another 2.2 million deaths, increasing the risk of cardiovascular and other diseases [1].

The fundamental, prospective 20-years Framingham Heart Study shows the importance of association between the diabetes and the cardiovascular disease. The diabetes is one of risk factors of cardiovascular events, and it also doubles the age-related risk of cardiovascular disease in men, and triples in women [3]. The diabetes was the main independent risk factor of cardiovascular diseases, even after the relative risk was adjusted on such cofounders as age, arterial hypertension, smoking, hypercholesterolemia, and left ventricular hypertrophy. 
The starting point for a good struggle against diabetes is its early diagnostic. As longer person lives with hidden hyperglycemia without diabetes verification and treatment, the harder will be complications and mortality from this disease. Diabetes particularly, type 2 , is completely asymptomatic, and frequently manifests with diabetic macroangiopathies, causing the CVR and the mortality from cardiovascular diseases. There are data that the development of cardiovascular complications of diabetes is caused by endothelial dysfunction that has a huge impact [4].

Nowadays, the search of sensitive and reliable biomarkers that simultaneously determines the risk of diabetes and the endothelial dysfunction is in constant progress. One of the reliable biomarkers that reflect the presence of endothelial dysfunction and T2DM is the Fatty Acid Binding Protein 4 (FABP4).

The limited number of studies on this issue was carried out only as a part of study devoted cardiovascular events risk. In a prospective 15-year study with 3700 participants, Luc Djousse with coauthors founded higher level of the FABP4 in patients with subsequently developed T2DM compared with patients without diabetes [5]. In this study, as well as in similar ones, researchers find a relationship between increased level of this marker and insulin resistance, body mass index, and level of unesterified free fatty acids [6], [7].

The results of experimental study in mice show, that decreased FABP4 level improved glucose and fat metabolism, reduced insulin resistance, atherogenesis and atherosclerosis, increased mice survival. All aforementioned, justified the possibility of using FABP4, as a potential marker of diabetes risk and monitoring its effectiveness treatment [8].

The analysis of clinical and experimental studies demonstrated the interlinks of the FABP4 biomarker and T2DM, and a connection of the FABP4 and cardiovascular events. However, relationships between increased FABP4 and risks of diabetes estimated by FINDRISC scale and cardiovascular events risk by SCORE scale have not been studied early. Our study team determined further interest in studying this topic at the stage of pre-nosological diagnosis to prevent fatal and non-fatal adverse cardiovascular events. We suggest that study of pathogenetic links between FABP4, prediabetes, and vascular catastrophes will be useful for creating early therapeutic strategy.

\section{Purpose}

The importance of the biomarker FABP4 to study, which determines the different risk of diabetes and cardiovascular events.

\section{Material and Methods}

\section{Respondents}

A prospective observational cross-sectional study of the population of Karaganda region was conducted simultaneously from May 2017 to December 2019. 389 respondents aged 18-65 took part in the study. There were 253 women $(65 \%)$ and 136 men $(34.7 \%)$. The study included questionnaires, anthropometry measuring height, weight, waist circumference (WC), calculating body mass index (BMI), measuring blood pressure (BP), determining fasting glucose, serum total cholesterol, and FABP4 biomarker.

The exclusion criteria were the following categories. Patients with the previous medical history of acute and decompensated heart diseases, including myocardial infarction, brain stroke confirmed by coronary angiography, and CT, and patients with chronic kidney disease and renal dysfunction based on glomerular filtration rate (GFR/CKD-EPI), $\leq 90 \mathrm{ml} / \mathrm{min} / 1,73 \mathrm{~m}^{2}$ were excluded from the study. Patients with previously diagnosed diabetes mellitus type 2 , and diabetes newly diagnosed by per oral glucose tolerance test, or those with plasma glucose at or above $11.1 \mathrm{mmol} / \mathrm{l}(200 \mathrm{mg} / \mathrm{dl})$ were excluded from the study. Pregnant women, people with severe mental and oncological diseases were excluded from the study.

Patients were divided into groups with diabetes risk (low and high) and control group. The control group consisted of 15 people aged 18-30, without any concomitant diseases.

Before the study, all respondents filled informed consent.

\section{The ethical approval}

The research team received approval from Local Ethical Committee of "Karaganda State Medical University," Karaganda, Kazakhstan, in accordance with principles of Declaration of Helsinki. The study protocol number is 309 from May 19, 2017.

\section{Measurement of serum FABP4 biomarker}

The study of the biomarker FABP4 serum level was carried out using a multiplex study, immunofluorescence method, and XMap technology. For this, Bioplex 3D instrument and reagent kit MILLIPLEX ${ }^{\circledast}$ Human cardiovascular disease panel (Millipore) were used.

\section{FINDRISC scale}

The risk of diabetes was assessed using the FINDRISC scale, questions of which included 
information on age, BMI, waist circumference(WC), a daily presence of at least 30 min of physical activity, taking antihypertensive drugs, data on the presence of diabetes in relatives, increased capillary glucose blood, and a daily consumption of fresh vegetables [9]. According to this scale, the examined persons with number of points below 7 had a low risk of type 2 diabetes (FINDRISC-1); from 7 to 11 points - a slightly increased risk of type 2 diabetes (FINDRISC-2); from 12 to 14 points - a moderate risk of T2DM (FINDRISC-3); and from 15 to 20 points - a high risk of T2DM (FINDRISC-4); more than 20 points - a very high risk of T2DM (FINDRISC-5). The FINDRISC gradation data reflect the likelihood of developing T2DM: FINDRISC-1 is than 1 out of 100 will have diabetes, FINDRISC-2 is than 1 out of 25 will have diabetes, FINDRISC-3 is than 1 out of 6 will have diabetes, FINDRISC-4 is than 1 out of 3 will have diabetes, and FINDRISC- 5 is than 1 out of 2 will have diabetes. Conventionally, all respondents were divided into two groups: The low and the high risk of T2DM. The low-risk group included respondents with a score of less than 11 (FINDRISC-1,2), and the highrisk group of T2DM - more than 12 (FINDRISC-3,4,5).

\section{Assessment CVR on the SCORE scale}

The CVR SCORE was calculated using an online calculator with free access, and using risk chart [10], [11]. Questions included age, gender, smoking issues, blood pressure (BP), and blood cholesterol in $\mathrm{mmol} / \mathrm{l}$. Persons with a score of $1 \%$ or less were included in a low CVR group (SCORE 1), more than $1 \%$ and $<5 \%$ - in a moderate CVR (SCORE 2), from $5 \%$ to $10 \%$ - in a high CVR (SCORE 3 ), over $10 \%$ - in a very high risk (SCORE 4). Conventionally, patients were divided into two groups: The low and high CVR (CVR). The low CVR group consisted of respondents with a score of less than 1\% (SCORE 1), the high CVR group was a score over $1 \%$ (SCORE 2,3,4). This scale also allows to assess the risk of person death from cardiovascular diseases over the next 10 years.

\section{Statistical analysis}

The data were tested for normal distribution using Kolmogorov-Smirnov test, the distribution was not normal. Variables not normally distributed were log-transformed. Statistical significance of differences between groups was evaluated using non-parametric Mann-Whitney U-test. Differences were considered statistically significant at $p<0.05$. The relationship of biomarker with the risk of diabetes and CVR was assessed using the Spearman correlation coefficient. The FABP4 associations with diabetes risk and CVR were calculated using a binary logistic regression model. The biomarker was tested in an unadjusted and adjusted model. Variables were logistically transformed before construction of regression model. Polynomial regression was used, studying factors affecting on the CVR in patients with diabetes risk. Statistical analysis was performed using IBM SPSS Statistics, 22.0. Results were considered statistically significant at $p<0.05$.

\section{Results}

The low risk of T2DM (FINDRISC-1) was found in $166(42.67 \%)$ respondents, the slightly increased risk (FINDRISC-2) was found in $129(33.16 \%)$, the moderate risk (FINDRISC-3) in 33 (8.48\%), the high risk (FINDRISC-4) in $37(9.51 \%)$, the very high risk (FINDRISC-5) - in $9(2.31 \%)$ people, and the control group was $15(3.85 \%)$ respondents. Thus, $295(75.83 \%)$ respondents were included in the lowrisk group of T2DM (FINDRISC-1,2), and $79(20.3 \%)$ respondents were included in the high-risk group of T2DM (FINDRISC-3,4,5) (Table 1).

In the high-risk group of diabetes, the median values of respondents' age were significantly higher than median age in the low-risk group. Thus, median of respondents' age in the high-risk group was 62 years old (Q25; Q75-58.0; 63.0), in the low-risk group was 56 years (Q25; $Q 75-46.0 ; 60.0 ; p=0,001)$.

Anthropometric characteristics of respondents, such as weight, BMI, WC in the group of respondents with the high risk of T2DM, were significantly higher than corresponding parameters in the low-risk group. In the low-risk group of T2DM, median of weight was $71.0 \mathrm{~kg}$ (Q25; Q75-63.0; 81.2), BMI was $26.13 \mathrm{~kg} / \mathrm{m}^{2}$ (Q25; Q75-23.65; 29.05), and WC was $89.0 \mathrm{~cm}$ (Q25; Q75-81.0; 96.0), while in respondents with the high risk of T2DM, these indicators were: Weight - 85.8 kg (Q25; Q75-78.0; 94.0), BMI - $33.2 \mathrm{~kg} / \mathrm{m}^{2}$ (Q25; Q75-30.06;

Table 1: Baseline characteristics of respondents with the diabetes risk and cardiovascular risk

\begin{tabular}{|c|c|c|c|c|c|c|c|}
\hline \multirow[t]{2}{*}{ Variables } & \multicolumn{3}{|c|}{ Low risk of T2DM (FINDRISC-1,2) $(n=295)$} & \multicolumn{3}{|c|}{ High risk of T2DM (FINDRISC-3,4,5) (n=79) } & \multirow[t]{2}{*}{$\mathrm{p}$-value } \\
\hline & Q25 & $\mathrm{Me}$ & Q75 & Q25 & $\mathrm{Me}$ & Q75 & \\
\hline Age, years & 46.0 & 56.0 & 60.0 & 58.0 & 62.0 & 63.0 & 0.001 \\
\hline Weight, kg & 63.0 & 71.0 & 81.2 & 78.0 & 85.8 & 94.0 & 0.000 \\
\hline Height, cm & 158.0 & 164.0 & 172.0 & 156.0 & 160.0 & 163.0 & 0.000 \\
\hline $\mathrm{BMI}^{*}, \mathrm{~kg} / \mathrm{m}^{2}$ & 23.65 & 26.13 & 29.04 & 30.06 & 33.25 & 37.04 & 0.000 \\
\hline WC, $\mathrm{cm}$ & 81.0 & 89.0 & 96.0 & 97.0 & 104.0 & 109.0 & 0.000 \\
\hline Systolic BP, mm of mercury & 110.0 & 120.0 & 140.0 & 125.0 & 140.0 & 160.0 & 0.000 \\
\hline Diastolic BP, $\mathrm{mm}$ of mercury & 70.0 & 80.0 & 90.0 & 80.00 & 90.0 & 100.0 & 0.000 \\
\hline Glucose, $\mathrm{mmol} / \mathrm{l}$ & 5.1 & 5.4 & 5.7 & 5.4 & 5.90 & 6.80 & 0.000 \\
\hline Cholesterol, $\mathrm{mmol} / \mathrm{l}$ & 4.19 & 5.09 & 6.15 & 4.68 & 5.53 & 6.54 & 0.000 \\
\hline SCORE, \% & 0.46 & 0.89 & 3.09 & 1.56 & 2.52 & 4.01 & 0.000 \\
\hline
\end{tabular}


33.25), WC - $104.00 \mathrm{~cm}(\mathrm{Q} 25 ; \mathrm{Q} 75-97.0 ; 109.0)$ (Table 1).

The level of the blood pressure, both systolic and diastolic, was higher among respondents with the high risk of T2DM. Significant differences were found by the level of glucose and cholesterol in respondents with the high risk of T2DM, compared with similar parameters of the low-risk group. Not only anthropometric and biochemical parameters was greater in the high diabetes risk group but also CVR points (\%) by SCORE scale. Accordingly, in high diabetes risk group the median of SCORE points comprised $2.52 \%$ and was regarded as a high CVR.

Issues were found that determine the risk of T2DM on the FINDRISC scale, so in the group with the high risk $55(69.62 \%)$ of respondents used antihypertensive therapy three folds often compared to the low-risk group. Consequently, in the low-risk group antihypertensive therapy was used among only $65(20.96 \%)$ respondents $(95 \% \mathrm{Cl} 0.586 ; 0.374 ; \mathrm{p}=$ 0.005 ). A daily 30 -min physical activity had no significant differences, and it was quite high in both studied groups. In the group with a high risk of T2DM, presence of a daily physical activity was noted at $71(89.87 \%)$ of respondents, and in the group with the low risk of T2DM the number was 278 (89.67\%); (95\% Cl 0.077; 0.073; $p=0.9$ ). A daily consumption of vegetables was slightly higher among respondents with the low risk of T2DM, and their number was 168 (54.18\%), compared with 40 $(50.63 \%)$ examined persons from the high-risk group $(95 \% \mathrm{Cl} 0.08 ; 0,15 ; p=0.4)$. The number of persons with burdened family history of T2DM was expectedly higher among respondents from the high risk of T2DM group and amounted to $38(48.10 \%)$. In the group with the low risk of T2DM, the share of them was $13.22 \%$, (95\% Cl 0.58; 0.46; $p=0.000)$.

A similar, statistically significant trend occurred in determining level of the FABP4. Hence, its level significantly prevailed in the high-risk group of T2DM
(Mean \pm SD; $53406.5 \pm 28443.9 \mathrm{pg} / \mathrm{ml} ; \mathrm{n}=79$; $p=0.000)$ compared with the low-risk group $(9510,8 \pm$ $4292,6 \mathrm{pg} / \mathrm{ml} ; \mathrm{n}=295)$, and the control group $(8161,1 \pm$ $3092,15 \mathrm{pg} / \mathrm{ml} ; \mathrm{n}=15$ ) (Figure 1).

Calculation of correlation coefficients reflected a presence of positive direct correlation between anthropometric, age-related factors, blood pressure, fasting glucose, cholesterol, and the risk of diabetes by FINDRISC scale. Studied marker of endothelial dysfunction - FABP4 positively correlated with age, weight, and fasting glucose. The positive strong and statistically significant correlation was found between FABP4 and BMI, WC. Association the FABP4 level with systolic and diastolic blood pressure, cholesterol was weaker than with previously listed parameters and it was statistically significant (Table 2).

Table 2: Binary logistic regression model. Unadjusted relative risk of T2DM and cardiovascular risk for the FABP4 marker

\begin{tabular}{|c|c|c|c|}
\hline Variables & B (se) & OR $(95 \% \mathrm{Cl})$ & $p$-value \\
\hline FABP4 (FINDRISC) & $0,238(0,999)$ & $4,330(2,717-6,901)$ & 0,000 \\
\hline FABP4 (SCORE) & $0,236(0,771)$ & $2,430(2,161-5,443)$ & 0,000 \\
\hline
\end{tabular}
$(r=0,594 ; p=0,000), \operatorname{CVR}(r=0,242 ; p=0,000)$ and the level of the FABP4 marker was established. A positive direct average statistically significant correlation reflects that the level of the FABP4 marker increased with the increased risk of T2DM on the FINDRISC scale, and CVR on the SCORE scale (Table 3).

FABP4 level also correlated with weight $(r=0,350 ; p=0,000)$, BMI $(r=0,475 ; p=0,000)$, and WC $(r=0,387 ; p=0,000)$.

Binary logistic regression model built for the FABP4 (OR: 4,330 [95\% Cl: 2,717-6,901]; $p=0,000$ ) showed relationship between FABP4 and risk of T2DM on the FINDRISC scale, as well as presence of relationship between the marker being studied and the CVR (OR: 2.430 [95\% Cl: 2.161-5.443]; $p=0,000$ ) (Table 2).
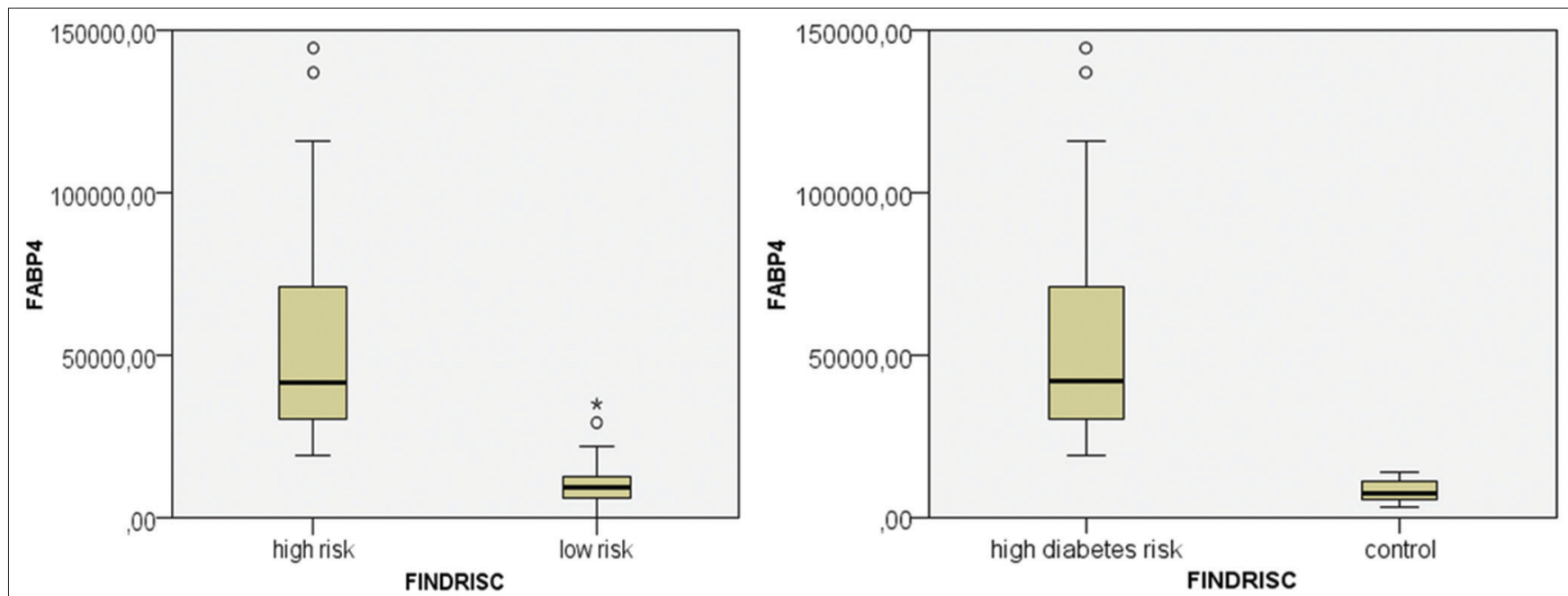

Figure 1: Level of the FABP4, depending on the different risk of diabetes. Mean values comparison of FABP4 in the different groups; significant $p=0.000$ 
Table 3: Correlation between the FABP4 level, glucose, cholesterol, anthropometric indicators; the risk of T2DM on the FINDRISC scale and cardiovascular risk on the SCORE scale. Spearman's correlation coefficient $(r)$

\begin{tabular}{ll}
\hline Variables & FABP4 pg/ml in the risk group T2DM - $(r)$ \\
\hline FABP4, pg/ml & - \\
Age, years & $0,231^{* *}$ \\
Weight, $\mathrm{kg}$ & $0,350^{* *}$ \\
Height, $\mathrm{cm}$ & $-0,253^{* *}$ \\
$\mathrm{BMI}, \mathrm{kg} / \mathrm{m}^{2}$ & $0,475^{* *}$ \\
WC, $\mathrm{cm}$ & $0,387^{* *}$ \\
Systolic BP, mm of mercury & $0,172^{* *}$ \\
Diastolic BP, mm of mercury & $0,117^{*}$ \\
Glucose, mmol/l & $0,265^{* *}$ \\
Cholesterol, mmol/l & $0,109^{*}$ \\
FINDRISC, (points) & $0,594^{* *}$ \\
SCORE, (\%) & $0,242^{* *}$ \\
\hline${ }^{* *} \mathrm{p}<0,0001,{ }^{*} \mathrm{p}<0,05 .{ }^{*}$ FABP4: Fatty acid binding protein, BMI: Body mass index, WC: Waist \\
circumference, BP: Blood pressure.
\end{tabular}

The adjusted risk by gender, age, and blood pressure according to the regression revealed a relationship between the risks of diabetes and CVR with an increased level of FABP4(Table 4).

Table 4: Binary logistic regression model. Adjusted relative risk of T2DM and CVR for the FABP4 marker by gender, age, level blood pressure

\begin{tabular}{lccc}
\hline Variables & $\mathrm{B}(\mathrm{se})$ & $\mathrm{OR}(95 \% \mathrm{Cl})$ & $\mathrm{p}$-value \\
\hline FABP4 (FINDRISC) & $1,210(0,118)$ & $3,330(0,447-3,37)$ & 0,000 \\
FABP4 (SCORE) & $0,752(0,608)$ & $2,121(0,644-6,988)$ & 0,000 \\
\hline \multicolumn{2}{l}{ Dependent variable is the risk of diabetes on the FINDRISC scale and cardiovascular risk on the SCORE }
\end{tabular}
Dependent variable is the risk of diabetes on the FINDRISC scale and cardiovascular risk on the SCORE scale compared to others.

We found factors affecting on the CVR in patients with the risk of T2DM. Indicators such as a total score on the FINDRISC scale, BMI, WC, and fasting glucose were established. Thus, an increase in the FINDRISC scores increases the CVR by 2.2 times, an increase of glucose level increases the CVR by 2.06 times, an increase of BMI by 1.2 times, and of WC by 1.0 times (Table 5).

Table 5: Polynomial regression. Factors affecting on the cardiovascular risk on the SCORE scale in patients with the risk of T2DM

\begin{tabular}{llll}
\hline Variables & $\mathrm{B}(\mathrm{se})$ & $\mathrm{OR}(95 \% \mathrm{Cl})$ & $\mathrm{p}$-value \\
\hline FINDRISC & 0,826 & $2,284(1,472-3,542)$ & 0,000 \\
Glucose, mmol/l & 0,726 & $2,067(1,097-3,898)$ & 0,025 \\
BMI, $\mathrm{kg} / \mathrm{m}^{2 *}$ & 0,223 & $1,249(0,660-2,365)$ & 0,04 \\
WC, cm & 0,040 & $1,041(0,968-1,119)$ & 0,02 \\
\hline *B
\end{tabular}

${ }^{*}$ BMI: Body mass index, WC: Waist circumference.

\section{Discussion}

This prospective cross-sectional study allowed us to identify groups of patients with the different risk of T2DM on the FINDRISC scale, and the different CVR on the SCORE scale. In addition, data allowed to establish an increase of FABP4 level in both group in high-risks of diabetes and cardiovascular events.

The study results demonstrated a predominance of the moderate and high risk in the structure of T2DM. In Karaganda region one out of five respondents had the high risk of diabetes (FINDRISC-3,4,5), which accounted 20\%. Comparative characteristics of respondents with FINDRISC-3,4,5 in the Republic of Kazakhstan are comparable with results of similar epidemiological studies in Saudi Arabia $(10.7 \%)$ and Libya $(12.3 \%)$, but lower than in Norway (28.5\%), in Cuba (26.8\%), and Mexico (28.9\%) and also in similar study which was conducted in the Kazakhstan from 2014 to 2016 [12], [13], [14], [15]. The 10-years risk of T2DM in Saudi Arabia (29.4\%), Libya (32.2\%), and Hungary $(41.0 \%)$ significantly exceeded the same indicator in Kazakhstan [12], [13], [14], [15]. Pakistani immigrants in Norway had a maximum percentage of the high diabetes risk representing about 53\% [15]. Studies devoted evaluation of the of cardiovascular events risk by the SCORE scale in patients at the stage of prediabetes on the FINDRISC scale have not been conducted.

We revealed the prevalence anthropometric characteristics of median values with the highest values of weight, WC, BMI, and reflecting Grade 3 obesity in the high-risk group of T2DM, which is comparable with general statistics, and emphasizes their significance in the development of T2DM [16]. Correlation analysis determined a positive average correlation $(r=0.59)$ between FABP4 biomarker and different risk of T2DM. Therefore, with an increased in the level of this marker, the diabetes risk on the FINDRISC scale increases and reflects a presence of endothelial dysfunction in these patients at the stage of absence impaired carbohydrate metabolism, using standard techniques (fasting blood glucose, glucose tolerance test, and glycated hemoglobin). At the same time, an increase in the FABP4 biomarker level begins to increase significantly already in the FINDRISC 3 respondent group.

The FABP4 level correlated with such risk factors of T2DM as increased WC and BMI, which emphasizes the influence of these factors on the development of endothelial dysfunction and confirms importance of insulin resistance in its genesis. According to results of the study, the growth of systolic blood pressure, and level of the FABP4, reached higher values in the FINDRISC 3,4,5 group, which is similar with results of the previous studies and evidenced a presence of endothelial dysfunction [17], [18]. The risk of increasing FABP4 marker persists even after adjusting it for age, sex, and blood pressure, which indicates possibly a greater significance of carbohydrate metabolism disturbance in the development of endothelial dysfunction. Performed correlation between hyperglycemia, hypercholesterolemia, blood pressure, and FABP4 level indicates on a presence of vascular wall damage and start of cascade metabolic reactions in patients with diabetes risk.

The diabetes risk on the FINDRISC scale increases in 4.3-fold with an increase of the level FABP4, it is consistent with studies conducted previously, which prospectively proved a more frequent occurrence of T2DM in individuals with an increase level of endothelial dysfunction marker [5], [7], [16], [17], [19]. An increase of the level FABP4 and reaching its maximum values in the groups high and very high 
CVR was found, during studying the CVR in patients with the risk of T2DM.

A 12-year prospective study by Wing Sun Chow and coauthors reported that the FABP4 biomarker was a predictor of cardiovascular events in $182(9.9 \% \%)$ out of 1847 obese patients with a high level of the FABP4 cardiovascular events, with median observational period of 9.4 years [17]. Prognostic power of this marker was studied in combination with C-reactive protein, reflecting a presence of systemic inflammatory response.

The CVR using the Framingham scale was assessed in a prospective study named «Monitoring of Trends and Determinants in Cardiovascular Disease» (MONICA), conducted by Koenig et al. After 6.6 years of follow-up, the percentage of cardiovascular events was slightly lower and consisted of 191 (5.6\%) out of 3435 patients [20]. The previous researches also identified factors determining the CVR in these patients; they have a high $\mathrm{BMI}, \mathrm{WC}$, systolic BP, diastolic BP, hyperglycemia, insulin resistance index (HOMA), and dyslipidemia [17], [21]. Thus, we can say that the FABP4 biomarker is a predictor of diabetes risk and cardiovascular events.

\section{Conclusions}

1. The level of the FABP4 significantly increased in both group with high risks of diabetes and cardiovascular events. FABP4 was associated with such factors as increased WC, BMI, and fasting hyperglycemia.

2. The established relationship between the level of FABP4 and the risks of diabetes and cardiovascular events evidences, its increase with rising points on the FINDRISC and SCORE scales, and reaches a peak in the high risk groups. Nevertheless, further monitoring of patients is necessary to determine the diagnostic importance of FABP4 as a predictor of the development cardiovascular events and diabetes mellitus.

3. The high level of the FABP4 in respondents with high risks of diabetes and cardiovascular events determines the importance of planning clinical strategy for the early identification of this category of people and targeted therapeutic effects, which will prevent the development of T2DM with a progression of endothelial dysfunction and reduce the further cardiovascular events.

\section{References}

1. International Diabetes Federation. IDF Diabetes Atlas. $8^{\text {th }}$ ed. Brussels, Belgium: International Diabetes Federation; 2017.
Available from: http://www.diabetesatlas.org. [Last accessed on 2019 Jan 23].

2. The National Register of the Republic of Kazakhstan; 2018. Available from: http://www.rcrz.kz/files/sbornik/sbornik_2018converted.pdf. [Last accessed on 2020 Mar 20].

3. Kannel W, McGee D. Diabetes and cardiovascular risk factors: The Framingham study. Circulation. 1979;59(1):8-13. https:// doi.org/10.1161/01.cir.59.1.8

PMid:758126

4. Yang Z, Shen Y, Shen W, Pu L, Meng H, Zhang R, et al. Elevated glycated albumin and reduced endogenous secretory receptor for advanced glycation endproducts levels in serum predict major adverse cardio-cerebral events in patients with T2DM and stable coronary artery disease. J Am Coll Cardiol. 2015;66(16):20-5. https://doi.org/10.1016/j.jacc.2015.06.316

5. Djousse L, Khawaja O, Bartz M, Biggs M, Ix J, Zieman S, et al. Plasma fatty acid-binding protein 4 , nonesterified fatty acids, and incident diabetes in older adults. Diabetes Care. 2012;35(8):1701-7. https://doi.org/10.2337/dc11-1690 PMid:22584136

6. Xu A, Wang Y, Xu J, Stejskal D, Tam S, Zhang J, et al. Adipocyte fatty acid-binding protein is a plasma biomarker closely associated with obesity and metabolic syndrome. Clin Chem. 2006;52:405-13. https://doi.org/10.1373/clinchem.2005.062463 PMid: 16423904

7. Annette W, Aimin X, Pak C, Nelson M, Yu Wang, Carol H, et al. Serum adipocyte fatty acid-binding protein as a new biomarker predicting the development of type 2 diabetes: A 10 -year prospective study in a Chinese cohort. Diabetes Care. 2007;30(10):2667-72. https://doi.org/10.2337/dc07-0413 PMid:17620449

8. Jeffrey B, Kazuhisa M, Makowski L, Babaev V, Fazio S, Linton M, et al. Combined adipocyte-macrophage fatty acid-binding protein deficiency improves metabolism, atherosclerosis, and survival in apolipoprotein E-deficient mice. Circulation. 2004;110(11):149298. https://doi.org/10.1161/01.cir.0000141735.13202.b6 PMid: 15353487

9. Alssema M, Feskens E, Bakker S, Gansevoort R, Boer J, Heine $\mathrm{R}$, et al. Finnish questionnaire reasonably good predictor of the incidence of diabetes in the Netherlands. Ned Tijdschr Geneeskd. 2008;152(44):2418-24.

PMid: 19055143

10. The Score Calculator; 2019. Available from: https://www. medsoftpro.ru/kalkulyatory/kalkulyator-score.html. [Last accessed on 2020 Jan 13].

11. Piepoli MF, Hoes AW, Agewall S, Albus C, Brotons C, Catapano Al, et al. 2016 European guidelines on cardiovascular disease prevention in clinical practice: The sixth joint task force of the European society of cardiology and other societies on cardiovascular disease prevention in clinical practice (constituted by representatives of 10 societies and by invited experts): Developed with the special contribution of the European association for cardiovascular prevention and rehabilitation (EACPR). Eur J Prev Cardiol. 2016;23(11):1-96. https://doi.org/10.1007/s12529-016-9583-6 PMid:27353126

12. Abduelkarem A, Sharif S, Hammrouni A, Aldouibi S, Albraiki W, Shareif $\mathrm{H}$. Risk calculation of developing type 2 diabetes Libyan adults. Pract Diabetes Int. 2009;26:148-51. https://doi. org/10.1002/pdi.1359

13. Mohieldein A, Mohieldein H, Alzohairy M, Hasan M. Risk estimation of Type 2 diabetes and dietary habits among adult Saudi non-diabetics in central Saudi Arabia. Glob J Health Sci. 2009;3:123-33. https://doi.org/10.5539/gjhs.v3n2p123

14. Naranjo A, Rodríguez A, Llera R. Diabetes risk in a Cuban primary care setting in persons with no known glucose 
abnormalities. MEDICC Rev. 2013;15(2):16-9. https://doi. org/10.37757/mr2013v15.n2.4

PMid:23686250

15. Sheryazdanova D, Ye L, Turmukhambetova A, Turgunova L. Assessment of the ten-year risk of developing T2DM in the Karaganda region: Population cross-sectional study. Med Ecol. 2016;2:64-8.

16. Daousi C, Casson I, Gill G, MacFarlane I, Wilding J, Pinkney J. Prevalence of obesity in type 2 diabetes in secondary care: Association with cardiovascular risk factors. Postgrad Med J. 2006;82(966):280-4. https://doi.org/10.1136/pmj.2005.039032 PMid:16597817

17. Chow W, Tso A, Xu A, Yuen M, Fong C, Lam T, et al. Elevated circulating adipocyte-fatty acid binding protein levels predict incident cardiovascular events in a community-based cohort: A 12-year prospective study. J Am Heart Assoc. 2013;2(1):e004176. https://doi.org/10.1161/jaha.112.004176 PMid:23525430

18. Ota H, Furuhashi M, Ishimura S, Koyama M, Okazaki Y, Mita T, et al. Elevation of fatty acid-binding protein 4 is predisposed by family history of hypertension and contributes to blood pressure elevation. Am J Hypertens. 2012;25(10):1124-30. https://doi. org/10.1038/ajh.2012.88

PMid:22717543

19. Djousséa L, Gazianoa J. Plasma levels of FABP4, but not FABP3, are associated with increased risk of diabetes. Lipids. 2012;47(8):757-62. https://doi.org/10.1007/ s11745-012-3689-7

PMid:22706792

20. Koenig W, Lowel H, Baumert J, Meisinger C. C-reactive protein modulates risk prediction based on the Framingham score: Implications for future riskassessment: Results from alarge cohort study in Southern Germany. Circulation. 2004;109(11):1349-53. https://doi.org/10.1161/01.cir.0000120707.98922.e3 PMid: 15023871

21. Eynatten M, Breitling L, Roos M, Baumann M, Rothenbacher D, Brenner $\mathrm{H}$, et al. Circulating adipocyte fatty acid-binding protein levels and cardiovascular morbidity and mortality in patients with coronary heart disease: A 10-year prospective study. Arterioscler Thromb Vasc Biol. 2012;32(9):2327-35. https://doi. org/10.1161/atvbaha.112.248609

PMid:22679309 